Vol.02/ No. 03

Pages: $121-127$

https://www.irojournals.com/iroiip/

DOI:_https://doi.org/10.36548/jiip.2020.3.001

\title{
Posed Inverse Problem Rectification Using Novel Deep Convolutional Neural Network
}

\author{
Dr. T. Vijayakumar, \\ Professor, \\ Department of ECE, \\ GNIT, Hyderabad, India. \\ Email id: vishal_16278@yahoo.co.in
}

\begin{abstract}
The proposed paper addresses the inverse problems using a novel deep convolutional neural network (CNN). Over the years, regularized iterative algorithms have been observed to be the standard approach to address this issue. Though these methodologies give an excellent output, they still impose challenges such as difficulty of hyper parameter selection, increasing computational cost for adjoint operators and forward operators. It has been observed that when the normal operator of the forward model is seen to be a convolution, unrolled iterative methods take up the CNN form. In view of this observation we have proposed a methodology which uses $\mathrm{CNN}$ after direct inversion to find the solution for convolutional inverse problem. In the first step the physical model of the system is analyzed using direct inversion. However, this leads to artifacts which are then removed using a combination of residual learning and multi-resolution decomposition in CNN. The results show that the performance of the proposed work outperforms other algorithm and requires a maximum of 1 second to reconstruct an image of high definition.
\end{abstract}

Keywords: Deep Convolutional Neural Network; artifacts; image processing; convolutional inverse problem

\section{Introduction}

During the past few years, a number of methodologies based on iterative reconstruction have been proposed to address the inverse problem faced which includes interpolation, de-convolution and de-noising [1]. There has been significant improvement in the image quality along with limited computational complexity due to compressed sensing and similar regularizes in the algorithms. This improvement has proven to be influential in the biomedical imaging field such as X-Ray computed tomography and Magnetic Resonance Imaging (MRI) [2]. Hence there is a trade-off between acquisition time and noise. In general, long acquisitions result in patient harm (in radiation-based methodologies) patient discomfort and motion artifacts while short acquisition will result in degrading the quality of images. In the recent years, deep learning is being used as an excellent tool to develop a promising framework which gives an excellent base for image segmentation and classification [3-4]. Also, regression based neural network have also resulted in excellent results when dealing with the inverse problem addressing issues such as interpolation, signal recovery, artifact reduction, deconvolution [5] and signal denoising [6].

In neural networks, the classic multilayer perception involves the use of arbitrary matrix multiplication in the different layers of the network. However due to the restriction on performing convolution, there is decrease in the need for learning more number of parameters. To solve this problem, researchers begun investigating deep learning networks [7] and the conventional iterative approaches, to provide a correlating link between the two methods. In [8] the authors analyzed the similarity between ISTA algorithm and proposed a neural network which comprised of shared layers resulting in fast approximated sparse coding. Similarly in [9] the authors proposed the use of iterative gradient descent inferences to solve the problem. Deep convolutional learning based on PeronaMalik was proposed in [10] which uses filters for diffusion gradient resulting in overcoming the general inversion problem. A relationship between deep neural network and penalized least squares methods was focused by the 
Journal of Innovative Image Processing (JIIP) (2020)

Vol.02/ No. 03

Pages: $121-127$

https://www.irojournals.com/iroiip/

DOI:_https://doi.org/10.36548/jiip.2020.3.001

authors in [11]. Though the authors of described works designed the algorithm in order to solve this issue, it was based on the assumptions that filters when incorporated in Perona Malik scheme learned, they increased the performance of the system thereby learning shrinkage function and number of filters. The proposed work explores the relationship between iterative optimization and $\mathrm{CNN}$ addressing one particular inverse problem. The problem addressed is that of normal operator which is associated with the forward model.

Deconvolution and denoising are the basic composition of the class and will also include diffraction tomography, $\mathrm{X}$-ray CT and MRI. Hence in order to solve this inverse problem, we propose a deep convolutional neural network that combines approximate and fast solver. In this paper we have used filtered back projection for CTreconstruction and for adaptive learning CNN is also implemented [12]. Since FBP-C reconstruction is used, it is also possible to make use of real data, without the need for storing data in the oracle knowledge. Both real and synthetic CT data results show positive quantitative measures when compared to other regularized iterative reconstruction. It is also observed that the images reconstructed used this methodology preserves the texture better when compared with other methods.

\section{Proposed Methodology}

Researchers have found that iterative methods which use pointwise non-linearities and filtering on the inverse problem have proved to be successful. Hence $\mathrm{CNN}$ is also recommended to be provide a compatible solution for the same and we have proposed a novel solution known as FBP-C Algorithm. This algorithm makes use of discretized [13] FBP-C as the input of the neural network. Here it is trained to bring a ground truth image based on the result of FBP-C. Though this methodology is suitable for all types of inverse problems in normal-convolution, in this paper work, we concentrate on CT reconstruction.

\subsection{FBP-C}

Filtered Back Projection (FBP) is performed initially before the image is sent to the CNN which can be used to directly regress information, thereby making the learning process simplified. The proposed methodology encapsulates our perspective of understanding the inverse problem such that we provide an appropriate input to CNN [14]. A good example for this scenario is the reconstruction of CT where the input is a sonogram and a traditional CNN will require change between the Cartesian and Polor coordinates. However when the proposed algorithm is used, this process can be avoided completely. It should also be noted that the proposed algorithm proves to be efficient for CT [15].

\subsection{Design of Deep CNN}

To address the inverse problem, we have used a unique CNN architecture which doesn't simply imitate the iterative methods of the system. The architecture embodies a number of properties which are as follows:

- Implementation Details: There are 2 important changes that are added to the U-net. First is that the last layer of the image is replaced with a convolutional layer Hence this will decrease the output image into 
https://www.irojournals.com/iroiip/

DOI:_https://doi.org/10.36548/jiip.2020.3.001

a single channel instead of 64 channels [16]. Second, zero-paddings are used in order to prevent degradation of the image size. Both the changes are essential as the architecture of U-net will lead to two channels namely background and foreground.

- Residual learning: On modifying the original U-net, a connection between the output and input is skipped indicating that this difference between output and input can be learnt by the network. Thus during the training process, it is possible to eliminate the problem of vanishing gradient, thereby improving the performance of the system.

- Multi-channel Filtering: At every layer, multichannel filters are used by the U-net to ma the features in every layer. In general, these filters can be used to increase the network's expressive power. Moreover, these channels make use of analog in iterative methods. This includes the use of wavelet coefficient vector 'a' by dividing it in such a way that it can be used by all the channels based on the sub-band of the wavelet. Similarly, channels can also be created in the form of split variables as seen in ADMM.

- Multi-level Decomposition: in order to ensure that the middle layer filters are considerably bigger that the other layers, it is essential to employ a dyadic scale decomposition that is based an algorithm. This is crucial as the filters used relate to $\mathrm{H}^{*} \mathrm{H}$ such that it will have non-compact support. Hence inversion of the convolution $\mathrm{H}^{*} \mathrm{H}$ is not possible when the filter size is small and fixed. Multiresolution wavelets can also be used during this decomposition process in iterative approaches.

\subsection{Computational Complexity}

More than filtering, the cost of the proposed algorithm is predominately used by back projection. For a sonogram with dimension $\mathrm{MxV}$ and image of dimension $\mathrm{NxN}, \mathrm{O}\left(\mathrm{N}^{2} \mathrm{MV}\right)$ is the cost of back projection, as far as worst-case scenario is taken into consideration. However, it is possible to further reduce this cost by using fixed-size discretization kernel which will result in a projection scale of $\mathrm{O}\left(\mathrm{N}^{2} \mathrm{~V}\right)$. The basic operations that we use in CNN include addition, convolution, local maximum filtering, down sampling, up sampling and ReLU function. Since the kernel used is small, the operation count requires the use of more convolution function that is used in space domain. In particular, it is used for L layers, $\mathrm{R}$ filters per layer, $\mathrm{KxK}$ filters, $\mathrm{NxN}$ input and also to evaluate the cost of CNN such that it grows in terms of $\mathrm{O}\left(\mathrm{R}^{2} \mathrm{~K}^{2} \mathrm{~N}^{2} \mathrm{~L}\right)$. Based on the biases and size of filters, the storage area of the network will vary. Hence it can also be represented as $\mathrm{O}\left(\mathrm{LK}^{2} \mathrm{R}^{2}\right)$. Back propagation algorithm is used for training (similar to that of forward propagation).

\section{Experiments}

This section deals with the reconstruction of the sparse-view X-Ray. The proposed FBP-C algorithm is compared with the iterative reconstruction methodology. This method uses the FFT-based filtering in the convolutional structure. The proposed algorithm starts with viewing the sonogram on which FBP-C is to be computed and is then compared with the other methods based on the following criteria.

\subsection{Data Preparation}

In the proposed methodology, there datasets are used for evaluation purpose. Two of the datasets are obtained from digital forward model and the other dataset uses experimental results from the environment.

1. Biomedical dataset is collected to build 500 original CR images from nearby clinics. This database uses 729 pixels and are built using iradon Matlab function and can be further used by FBP-C. 
Journal of Innovative Image Processing (JIIP) (2020)

Vol.02/ No. 03

Pages: $121-127$

https://www.irojournals.com/iroiip/

DOI:_https://doi.org/10.36548/jiip.2020.3.001

2. More than 500 images of random location, size and intensity make up the synthetic dataset known as ellipsoid dataset. Similar to the previous dataset, it uses 729 pixels and are built using iradon Matlab function and can be further used by FBP-C.

3. 378 sinograms make up the experimental dataset which are real CT dataset. Here every sonogram will have 1493 pixels by 721 views. The sonograms are subsampled by factors of 20 and 7 corresponding to 50 and 143 views. In the case of the real data, factors of 14 and 5 were used to subsample, accounting for 52 and 142 views.

\subsection{Training Procedure}

1. FBP-C: A total of 475 training images are used for building the synthetic dataset of which 25 are test images. For real data, 327 images are used as images for training. Similarly a different set of training images are used for biomedical dataset which can be used to test data. A gap of 25 slices are said to exist between training and testing data that can be used by z-slices. An adjustment is done between the values of -500 and +500 . Full view FBP and low-view FBP are used as the output images and input images to train the CNN. It should be noted that the proposed methodology cannot provide access to oracle reconstruction but only to CT reconstruction.

2. TV Reconstruction: On a conclusive note, we address how training and testing data is done using the iterative methods. We have chosen the parameters for optimization performance to result in a fair comparison.

\section{Experimental Results}

Additive White Gaussian Noise is present in the output and the testing sets also have a decrease in views, representing the stability of the proposed methodology.

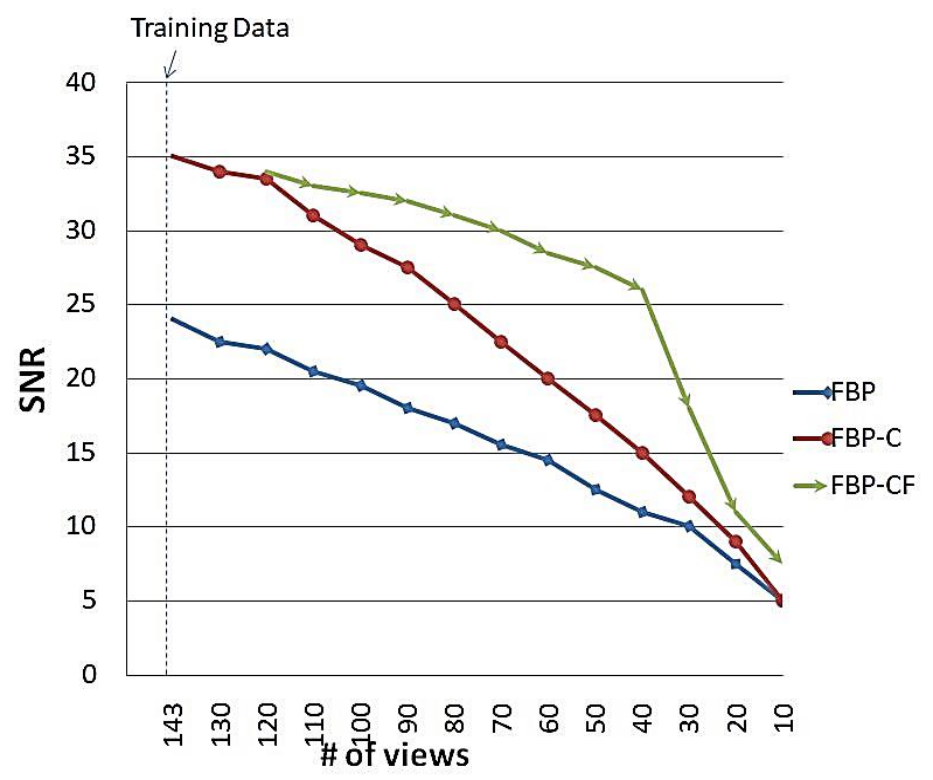


Vol.02/ No. 03

Pages: $121-127$

https://www.irojournals.com/iroiip/

DOI:_https://doi.org/10.36548/jiip.2020.3.001

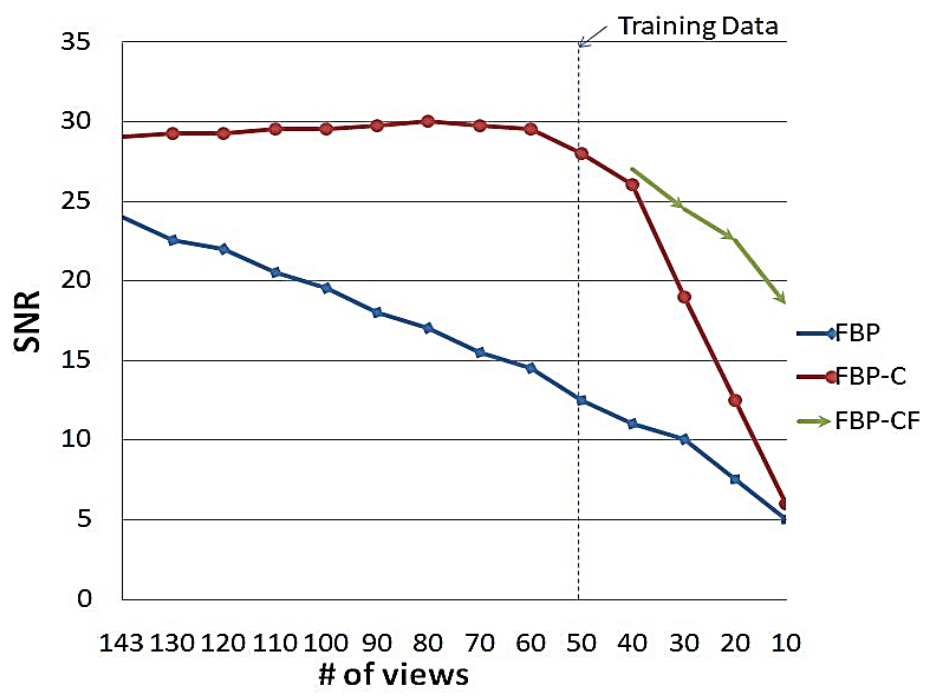

Fig.1. Number of view for training data of 143 views and 50 views

Varying the number of views a fixed set of views is used to train the network. Fig.1 shows two graphs that represent degradation of SNR based on the training set projection of 143 views with reduced number of views. This is further trained for a set of 10 more epochs with the help of the views count. Here the lower graph represents a similar graph that represents 50 views.

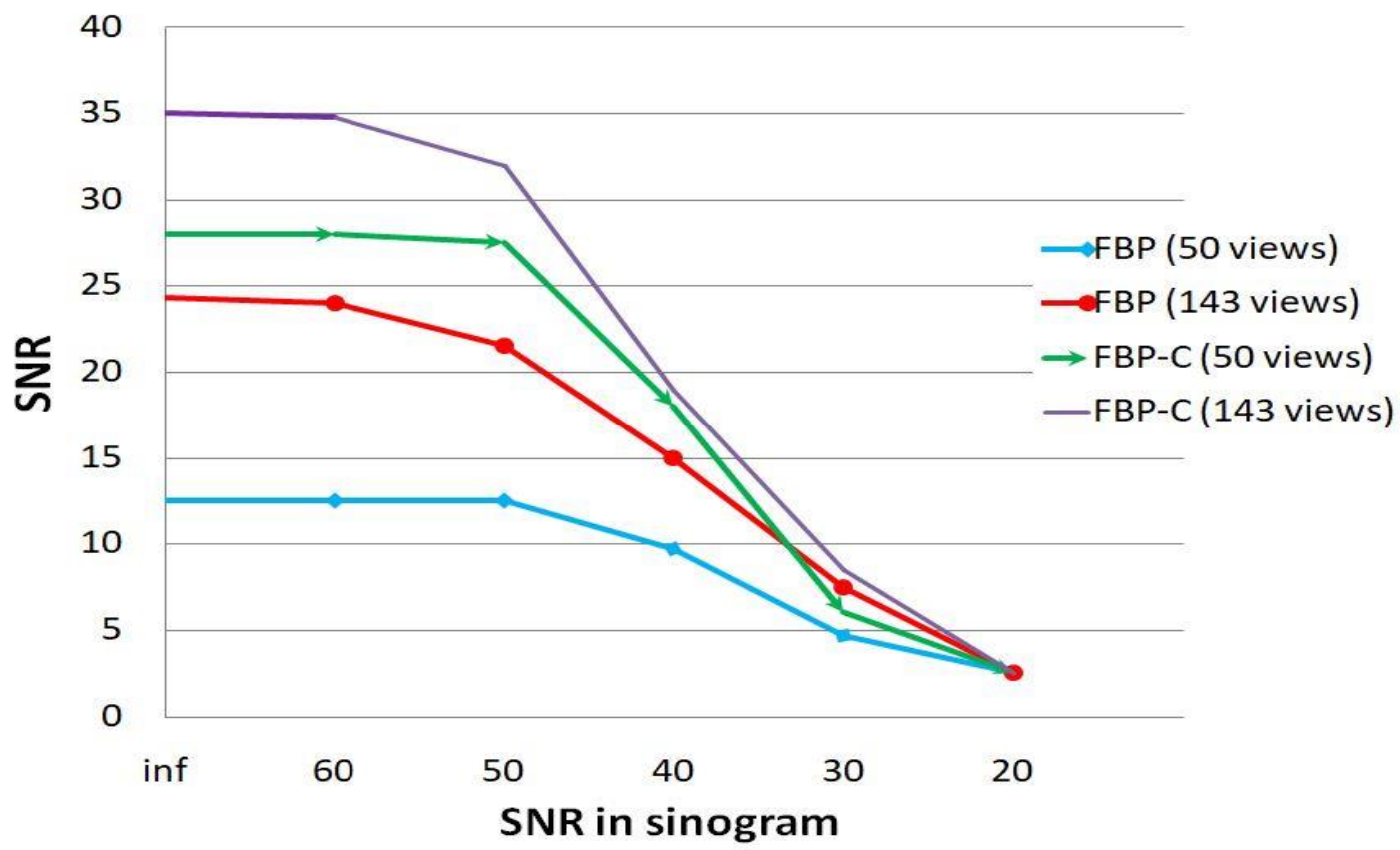

Fig.2. SNR of FBP and FBP-C for 50 and 143 views 
Journal of Innovative Image Processing (JIIP) (2020)

Vol.02/ No. 03

Pages: $121-127$

https://www.irojournals.com/iroiip/

DOI:_https://doi.org/10.36548/jiip.2020.3.001

The observed results indicate there is degradation in the performance of the network when tested with images other than that of the testing sets, which is still better than that of reconstruction of FBP. It is possible to reduce the gradation by fine-tuning the data using new environment. Similarly when projection views of 50 or 143 projection views are used to train the data without AWGN which is represented in Fig.1.b. Similarly Fig.2. represents the stable performance of FBP and FBP-C for 50 and 143 views. The performance of the system will decline gradually when the noise level is high. Based on these analysis, it can be observed that the range in which original training conditions can be broader than the pre-defined acceptable values.

\section{Conclusion}

The proposed work presents an effective way to address the inverse problems using deep convolution with particular focus on biomedical data. We have used FBP-C which combines CNN and FBP of which CNN uses residual learning and is built on U-net. A number of inverse problems in the field of biomedical (DT, MRI and CT) can be addressed using the proposed convolutional structure. We have also outlined a linear operator which acts as convolution in the normal operator. Thus CNN proves to be the apt fit to address the inverse problem. This research work shows satisfactory results on real data as well as synthetic data. Moreover, on training, it is observed that the computation time is less than a second for one image.

\section{References}

[1] Vijayakumar, T. (2019). Comparative Study Of Capsule Neural Network In Various Applications. Journal Of Artificial Intelligence, 1(01), 19-27.

[2] Vijayakumar, T. (2019). Neural network analysis for tumor investigation and cancer prediction. Journal of Electronics, 1(02), 89-98.

[3] Bindhu, V. (2019). Biomedical Image Analysis using Semantic Segmentation. Journal of Innovative Image Processing (JIIP), 1(02), 91-101.

[4] Vijayakumar, T., \& Vinothkanna, M. R. (2020). Mellowness Detection of Dragon Fruit Using Deep Learning Strategy. Journal of Innovative Image Processing (JIIP), 2(01), 35-43.

[5] M. G. McGaffin and J. A. Fessler, "Alternating dual updates algorithm for X-ray CT reconstruction on the GPU,” IEEE Trans. Comput. Imag., vol. 1, no. 3, pp. 186-199, Sep. 2015.

[6] M. T. McCann, M. Nilchian, M. Stampanoni, and M. Unser, "Fast 3D reconstruction method for differential phase contrast X-ray CT,” Opt. Exp., vol. 24, no. 13, pp. 14564-14581, Jun. 2016.

[7] D. A. Janeera and Sasipriya.S. "A Brain Computer Interface Based Patient Observation and Indoor Locating System with Capsule Network Algorithm" In International Conference on Image Processing and Capsule Networks (ICIPCN 2020), Springer, Thailand, 2020.

[8] Y. LeCun, Y. Bengio, and G. Hinton, “Deep learning,” Nature, vol. 521, pp. 436-444, May 2015.

[9] Ruth Anita Shirley D, Ranjani K, Gokulalakshmi Arunachalam, Janeera D.A., "Distributed Gardening System Using Object Recognition and Visual Servoing" In International Conference on Inventive Communication and Computational Technologies [ICICCT 2020], Springer, India, 2020.

[10] O. Russakovsky et al., "ImageNet large scale visual recognition challenge," Int. J. Comput. Vis., vol. 115 , no. 3, pp. 211-252, Dec. 2015.

[11] Jayashree, S. and D. A. Janeera. "Real-Time Fire Detection, Alerting and Suppression System using Live Video Surveillance.” (2016).

[12] R. Girshick, J. Donahue, T. Darrell, and J. Malik, "Rich feature hierarchies for accurate object detection and semantic segmentation," in Proc. IEEE Conf. Comput. Vis. Pattern Recognit., Jun. 2014, pp. 580-587.

[13] J. Long, E. Shelhamer, and T. Darrell, "Fully convolutional networks for semantic segmentation," in Proc. IEEE Conf. Comput. Vis. Pattern Recognit., Jun. 2015, pp. 3431-3440. 
Journal of Innovative Image Processing (JIIP) (2020)

Vol.02/ No. 03

Pages: $121-127$

https://www.irojournals.com/iroiip/

DOI:_https://doi.org/10.36548/jiip.2020.3.001

[14] O. Ronneberger, P. Fischer, and T. Brox, "U-Net: Convolutional networks for biomedical image segmentation,” in Proc. Int. Conf. Med. Image Comput. Comput.-Assist. Intervent, 2015, pp. 234241.

[15] H. Chen, Y. Zhang, W. Zhang, P. Liao, K. Li, J. Zhou, and G. Wang, "Low-dose CT via convolutional neural network," Biomed. Opt. Exp., vol. 8, no. 2, pp. 679-694, Feb. 2017.

[16] K. He, X. Zhang, S. Ren, and J. Sun, “Deep residual learning for image recognition,” in Proc. IEEE Conf. Comput. Vis. Pattern Recognit., Jun. 2016, pp. 770-778.

\section{Authors Biography}

Dr. T. Vijayakumar, is currently working as Professor, at Department of ECE, GNIT, in Hyderabad, India. His research areas include Image Analysis, Object tracking and Recognition, Image coding, enhancement, Filtering, Rendering, Restoration, Half-toning, Search, and Vision-based Human - Computer Interaction. 